Case Report

\title{
Endophthalmitis caused by Kocuria kristinae A Case Report
}

\author{
M Alles $^{1}$, D Wariyapola ${ }^{1}$, K Salvin $^{1}$, K Jayatilleke $^{1}$ \\ Sri Lankan Journal of Infectious Diseases 2020 Vol.10(2):146-149 \\ DOI: http://dx.doi.org/10.4038/sljid.v10i2.8281
}

\begin{abstract}
Kocuria kristinae is part of the microbiota of humans but can also act as an opportunistic pathogen causing invasive infection. Here we report a case of endophthalmitis in a previously healthy adult male presumably resulting from iatrogenic infection due to intra-vitreal administration of drugs for the management of macular oedema. Following trans pars plana vitrectomy, an aseptically obtained specimen of vitreous humor was processed for bacterial culture. A pure growth of $K$. kristinae was isolated which was identified via VITEK 2. The patient was treated with intra-vitreal vancomycin with subsequent improvement of his condition.
\end{abstract}

Keywords: Kocuria kristinae, endophthalmitis, vancomycin

\section{Introduction}

Kocuria kristinae is a Gram positive coccus, ubiquitous in nature and a commensal of the skin and mucous membranes of humans. It can also manifest as an opportunistic pathogen, particularly in patients who are immunocompromised. ${ }^{1}$ Nevertheless, only few cases of infection associated with this organism have been reported worldwide, ${ }^{2}$ resulting in a very limited amount of data concerning such infections. Here we present the first reported case of endophthalmitis caused by K. kristinae in Sri Lanka.

\section{Case report}

A previously healthy farmer aged 67 years presented to his local District General hospital with acute onset of blurring of vision in his right eye. Ophthalmological examination showed branch retinal vein occlusion causing macular oedema of the right eye. He was treated with 2 doses of intravitreal bevacizumab (trade name "Avegra") administered 3 months apart. Following the initial dose, he reported that the visual blurring of his right eye had improved.

${ }^{1}$ Sri Jayawardenapura General Hospital, Sri Lanka

Address for correspondence: Dr Mario Alles, Sri Jayawardenapura General Hospital, Sri Lanka

Telephone: +94774307125 E-mail:alles_mario@yahoo.co.uk iD https://orcid.org/0000-0003-3718-2146

Received 30 December 2019 and revised version accepted 29 July 2020

(c) (7)

use, distribution, and reproduction in any medium, provided the original author and source are credited. 
However, 5 days after the $2^{\text {nd }}$ dose, he developed acute visual blurring in his right eye. Ophthalmological examination on this occasion showed a hypopyon in his right eye with B scan features of reticular echogenic material within the vitreous humor. He was managed as endophthalmitis of the right eye for which intravitreal vancomycin, ceftazidime and moxifloxacin were administered followed by one week of treatment with topical antibiotics which included vancomycin and ceftazidime along with oral itraconazole.

Due to persistent visual impairment of the right eye, the following week he was presented to a tertiary care teaching hospital following which a trans pars plana vitrectomy was performed and a specimen of vitreous humor was directly plated on blood and chocolate agar plates for bacterial culture.

Circular, low convex, non-hemolytic white colonies which were $2 \mathrm{~mm}$ in diameter grew on both plates following overnight aerobic incubation. These produced a positive catalase test but a negative slide coagulase test. Gram stain showed Gram positive large round cocci in tetrads (Figure 1), depicting the Gram stain appearance of Micrococcus species. The isolate was tested for sensitivity to furazolidone and bacitracin using disk diffusion to confirm an identification of Micrococcus and was found to be resistant to both. However, a zone of inhibition (diameter of $16 \mathrm{~mm}$ ) was visible around a vancomycin disk, placed for the purpose of assessing the isolates sensitivity to the drug.

The isolate was identified via VITEK 2 as K. kristinae. However, the automated system failed to produce an antibiotic sensitivity

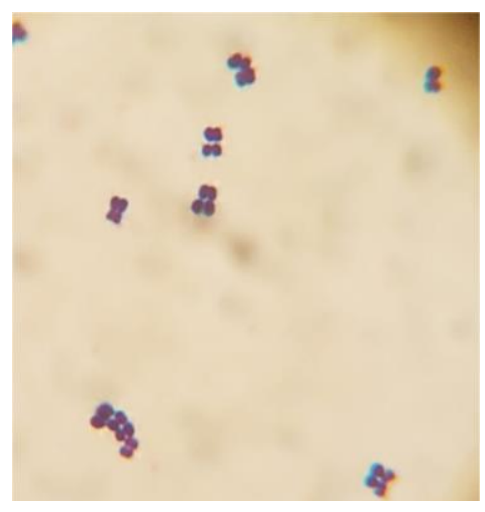

Fig. 1: Gram positive cocci in tetrads pattern.

Following surgery, a dose of intravitreal vancomycin and ceftazidime was administered to the patient followed by topical moxifloxacin which was planned to be continued for 2 weeks. At the time of writing, which was one week following surgery, he was undergoing clinical follow up and revealed an improvement in vision in his right eye.

\section{Discussion}

The genus Kocuria belongs to the family Micrococcaceae, suborder Micrococcineae, and order Actinomycetales. A total of 18 species have been described within the genus, out of which five (including $K$. kristinae) are known to be opportunistic pathogens. ${ }^{3}$

$K$. kristinae is a facultative anaerobe which is non-motile, catalase positive, coagulase negative, and gives a Gram stain appearance depicting Gram positive cocci arranged in tetrads (Figure 1), while producing pale cream non-hemolytic colonies on blood agar. ${ }^{2}$ Among immunocompromised patients, it is known to cause endocarditis and central venous catheter related bacteremia, while cholecystitis has been reported in an immunocompetent individual. ${ }^{4}$ Our patient was a healthy adult with no history of immune suppression. 
Few cases of endophthalmitis due to Kocuria species have been reported globally. In a five year study involving eight cases of Kocuria endophthalmitis ${ }^{5} 5$ were due to open globe injury. The rest followed cataract surgery and microbial keratitis with one having an endogenous cause. All eight patients were found to be immunocompetent. Iatrogenic vitreal infection is likely to have occurred in our patient following intravitreal bevacizumab. Studies have shown that a lapse in the aseptic technique during administration of injectable drugs into sterile spaces result in introducing infection into that space. ${ }^{6}$

The culture characteristics of our isolate along with the biochemical features and Gram stain appearance would result in a misidentification as either coagulase negative Staphylococcus or Micrococcus species. However, the isolate appeared completely resistant to both furazolidone and bacitracin, indicating that it is neither of them. Hence, VITEK 2 was used to identify it. Studies have shown conclusively that the currently used gram positive identification card in the VITEK 2 system correctly identifies isolates as K. kristinae. ${ }^{7}$

Standardized guidelines for antibiotic susceptibility testing do not exist for Kocuria species, and therefore breakpoints for staphylococci are used for this purpose. However, this can lead to misinterpretation of antibiotic susceptibility. ${ }^{5}$ Studies reveal highest susceptibility of $K$. kristinae to vancomycin, with high efficacy rates being shown. ${ }^{8}$ Due to static and dynamic barriers of the eye which impair the penetration of systemic and topical antibiotics, satisfactory levels in the vitreous can only be achieved if administered via the intravitreal route. ${ }^{9}$ Two doses of intravitreal vancomycin given 3-4 days apart has been shown to be effective in clearance of the organism, with therapeutic levels of vancomycin being maintained for up to a week following the first injection. One study found all second vitreous taps to be culture negative with the above protocol. ${ }^{10}$ Those who remain culture positive by this time would eventually need vitrectomy, due to bacterial sequestering or biofilm formation, rather than antibiotic resistance. ${ }^{11}$ Following vitrectomy, it is the usual practice to administer intravitreal antibiotics to ensure complete clearance of the organism. ${ }^{12}$ Even though topical antibiotics are not part of the treatment protocol of endophthalmitis, it is commonplace to administer such in order to ensure sterility of the anterior chamber in the postoperative period. ${ }^{13}$

In conclusion, $K$. kristinae, although only rarely a pathogen, can cause invasive infection such as endophthalmitis in a healthy adult. Accurate identification of the organism can be challenging due to certain features shared with coagulase negative staphylococci and Micrococcus species. Treatment of Kocuria endophthalmitis should consist of intra-vitreal vancomycin.

\section{Acknowledgement:}

We thank the patient and the family for consenting to the publication of the case details.

Conflict of interest: None declared.

\section{References}

1. Savini V, Catavitello C, Masciarelli G, et al. Drug sensitivity and clinical impact of members of the genus Kocuria. Journal of Medical Microbiology. 2010;59(12):1395-402. 
doi: https://doi.org/10.1099/jmm.0.021709-0

2. Dunn R, Bares S, David MZ. Central venous catheter-related bacteremia caused by Kocuria kristinae: Case report and review of the literature. Ann Clin Microbiol Antimicrob. 2011;24;10:31. doi: https://doi.org/10.1186/1476-0711-10-31

3. Hassan RM, Bassiouny DM, Matar Y. Bacteremia caused by Kocuria kristinae from Egypt: are there more? A case report and review of the literature. Case Rep Infect Dis 2016; Article ID 6318064. doi: https://doi.org/10.1155/2016/6318064

4. Tewari R, Dudeja M, Das AK, et al. Kocuria Kristinae in catheter associated urinary tract infection: A case report. J Clin Diagn Res. 2013;7(8):1692-3. doi: https://doi.org/10.7860/JCDR/2013/6077.3247

5. Dave VP, Joseph J, Pathengay A, et al. Clinical presentations, management outcomes, and diagnostic dilemma in Kocuria endophthalmitis. J Ophthalmic Inflamm Infect 2018; 8:21 doi: 10.1186/s12348-018-0163-6

6. Gargiulo DA, Mitchell SJ, Sheridan J, et al. Microbiological contamination of drugs during their administration for anesthesia in the operating room. Anesthesiology. 2016;124(4):785-94. doi: https://doi.org/10.1097/ALN.0000000000001041

7. Boudewijns M, Vandeven J, Verhaegen J. Vitek 2 Automated identification system and Kocuria kristinae. J Clin Microbiol. 2005;43(11):5832. doi: https://doi.org/10.1128/JCM.43.11.5832.2005

8. ŽivkovićZarić RS, Pejčić AV, Janković SM, et al. Antimicrobial treatment of Kocuria kristinae invasive infections: Systematic review. J Chemother. 2019;31(3):109-19. doi: https://doi.org/10.1080/1120009X.2018.1542551

9. Radhika M, Mithal K, Bawdekar A, et al. Pharmacokinetics of intravitreal antibiotics in endophthalmitis. Journal of Ophthalmic Inflammation and Infection. 2014; 10:4(1):22. doi: https://doi.org/10.1186/s12348-014-0022-z

10. Gan I, van Dissel JT, Beekhuis W, et al. Intravitreal vancomycin and gentamicin concentrations in patients with postoperative endophthalmitis. Br J Ophthalmol. 2001;85(11):1289-93. doi: http://dx.doi.org/10.1136/bjo.85.11.1289

11. Leung EH, Kuriyan AE, Flynn HW, et al. Persistently vitreous culture-positive exogenous bacterial endophthalmitis. Am J Ophthalmol. 2016;165:16-22. doi: https://doi.org/10.1016/j.ajo.2016.02.017

12. Clarke B, Williamson TH, Gini G, et al. Management of bacterial postoperative endophthalmitis and the role of vitrectomy. Surv Ophthalmol. 2018;63(5):677-93. doi: https://doi.org/10.1016/j.survophthal.2018.02.003

13. Cagini C, Piccinelli F, Lupidi M, et al. Ocular penetration of topical antibiotics: study on the penetration of chloramphenicol, tobramycin and netilmicin into the anterior chamber after topical administration: Ocular penetration of topical antibiotics. Clinical \& Experimental Ophthalmol. 2013; 41(7):644-647 doi: https://doi.org/10.1111/ceo.12087 